\title{
Ocular surface squamous neoplasia: a case series
}

\author{
Gholamhossein Yaghoobi', Abbas Hosseini Rad', \\ Saeed Reza Heydari², Malihe Nikandish' \\ 'Ophthalmology Department, Valiasr Hospital, Birjand University of Medical \\ Sciences, Birjand, Iran; ${ }^{2}$ Mashhad University of Medical Science, Mashhad, Iran
}

\begin{abstract}
Purpose: This study was conducted to show diverse clinical presentation of ocular surface squamous neoplasia with a rare presentation of caruncle squamous cell carcinoma (SCC).

Methods: All cases that had suspicious ocular lesion were included in the treatment study. A detailed history including demographic data was obtained. Clinical features regarding the type of lesion, location, and the involvement of cornea were evaluated. For all patients, excisional biopsy following "Shield's no-touch technique" with 3 to $4 \mathrm{~mm}$ margins of uninvolved tissue and cryotherapy at excisional margins was done. All specimens were sent for a histopathological evaluation. Topical chemotherapy (mitomycin C, $0.02 \%$ ) was used as an adjunctive therapy following surgical excision for large and diffuse ocular surface tumors. All patients were subjected to long-term regular follow-ups.

Results: Twenty-six patients (18 men and 8 women) with a mean age of 54.2 years were enrolled in this study. The results showed that the most common localization was bulbar conjunctiva (92\%). Nodular lesions (46.5\%) and SCC (57.5\%) were the most common clinical and histopathological features, respectively. We observed one rare case of primary SCC of the left caruncle in a 68-year-old man who had an asymptomatic medial canthal mass. Recurrence was found in two patients with SCC, one of them having an orbital extension.

Conclusion: The early suspicion of ocular surface neoplasia will be accomplished with a simple excision in most cases, leading to a favorable outcome except in severe progressive cases.
\end{abstract}

Keywords: caruncle, ocular surface squamous neoplasia, squamous cell carcinoma

\section{Introduction}

Ocular surface squamous neoplasia (OSSN) is a spectrum of pathology ranging from noninvasive intraepithelial dysplasia of the conjunctiva and cornea to invasive squamous cell carcinoma (SCC). ${ }^{1}$ The limbal stem cells are the origin of OSSN, which normally arise in the interpalpebral region and involved the bulbar conjunctiva, the cornea, or both structures. ${ }^{2}$

Correspondence: Malihe Nikandish, Valiasr Hospital, Ayatollah Ghaffari Ave., Birjand, Southern Khorasan Province, Iran.

E-mail: nikandishm@bums.ac.ir; malihenikandish@yahoo.com 
There are no consistent clinical criteria for distinguishing intraepithelial dysplasia from invasive SCC. The presence of feeder vessels, intrinsic vascularity, and a nodular lesion raise suspicion of invasive SCC. ${ }^{3}$

Male gender, temporal and superior locations, lack of corneal involvement, and papillomatous and nodular appearance were associated with higher-grade OSSN lesions in a study in the United States. ${ }^{4}$

While histopathology is the gold standard in the diagnosis of OSSN, high-resolution optical coherence tomography can be used to noninvasively detect the presence of OSSN in patients with coexisting ocular conditions. ${ }^{5}$

The standard modality for treatment of OSSN ranges from wide surgical excisions to "no-touch" technique and adjunctive cryotherapy. ${ }^{6}$

OSSN has a good prognosis; with the modern techniques, the recurrence rate is about $5 \%$ and regional metastasis is of $2 \%{ }^{6}$

This study describes diverse clinical presentation and treatment response in a series of patients attended our eye clinic.

\section{Methods}

All cases that had suspicious ocular surface lesions participated in the treatment study after submitting their inform consent. A detailed history including demographic data of age, sex, occupation, and HIV status of all the patients was obtained. Clinical features including the type of lesion, location, and the involvement of cornea were evaluated. For all patients, excisional biopsy following "Shield's no-touch technique" with 3 to $4 \mathrm{~mm}$ margins of uninvolved tissue and cryotherapy at excisional margins was done. Affected corneal epithelium was completely removed using absolute alcohol, with care taken to avoid the Bowman's layer. All specimens were sent for histopathological evaluation. Topical chemotherapy was used as an adjunctive therapy following surgical excision for large and diffuse ocular surface tumors. Topical chemotherapy regimen included mitomycin C (0.02\%) four times daily with cycles of two weeks on, two weeks off, and maximum two cycles until clinical resolution occurred. We prevented punctal stenosis and reduced systemic absorption by punctual plug placement. All patients had long-term regular follow-ups, and complete ocular surface examination and palpation of the regional lymph node were done at each visit.

\section{Results}

Twenty-six patients ( 18 men and 8 women) with a mean age of 54.2 years (range: 31-74 years) participated in this study. Table 1 shows the distribution of the lesions according to sex and age. The mean follow-up period was 20 months. All patients were negative for HIV. At presentation, the tumor involved the limbus and cornea in 15 eyes, forniceal conjunctiva in one eye, and no tarsal conjunctiva 
Table 1. Distribution of OSSN regarding the patients' age and sex.

\begin{tabular}{|l|l|l|l|}
\hline $\begin{array}{l}\text { Age } \\
\text { (years) }\end{array}$ & Men & Women & Recurrence \\
\hline$<30$ & 0 & 0 & 0 \\
\hline $31-40$ & 1 & 0 & 0 \\
\hline $41-50$ & 4 & 1 & 0 \\
\hline $51-60$ & 5 & 4 & 0 \\
\hline$>60$ & 8 & 3 & 2 \\
\hline Total & $\mathbf{1 8 ( 6 9 \% )}$ & $\mathbf{8 ( 3 1 \% )}$ & $\mathbf{2}$ \\
\hline
\end{tabular}

involvement. One patient had both temporal and nasal involvement in the right eye. Seven patients (27\%) presented with pterygium-like lesions, $12(46.5 \%)$ with nodular lesions (Fig. 1), and six (23.5\%) with gelatinous pattern. The pattern of distribution of OSSN lesions within the interpalpebral fissure of the ocular surface in this study was most commonly on the nasal side (57.5\%), followed in the order by temporal (27\%), superior (7.5\%), inferior (4\%), and caruncle (4\%), respectively. A total of $83.7 \%$ of subjects had both corneal and conjunctival involvement, while $16.3 \%$ had conjunctival involvement only. A total of $38.4 \%$ of subjects had dysplasia and $57.6 \%$ had SCC (Fig. 2). Localization and distribution pattern of the lesions, as well as clinical and histopathological features, are shown in Table 2.

Five patients received adjuvant topical chemotherapy following surgical excision because of the large size of lesions (Fig. 3). Recurrence was found in two patients with SCC, one of them having an orbital extension. This patient refused further intervention and died several months later due to this malignant disease.

We had one rare case of ocular surface neoplasia, a primary SCC of the left caruncle in a 68-year-old man (Fig. 4) who presented with asymptomatic medial canthal mass and underwent wide-margin surgical excision and adjuvant topical chemotherapy.

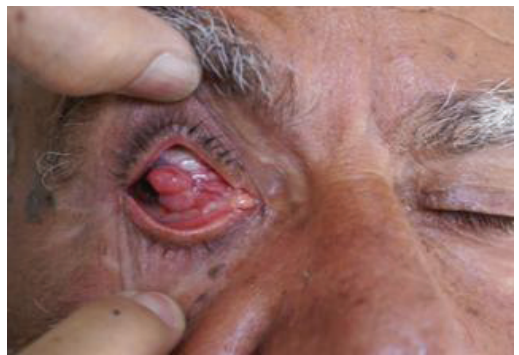

Fig. 1. Nodular OSSN.

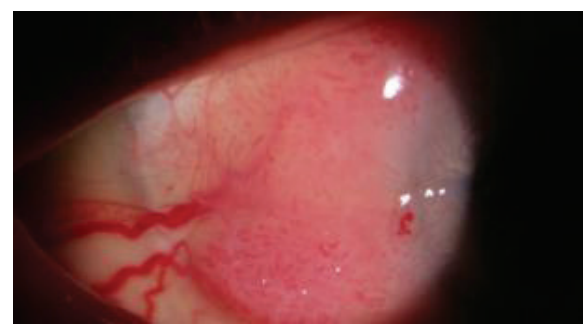

Fig. 2. Limbal SCC with prominent intrinsic vascularity and feeding vessel. 
Table 2. Distribution of OSSN regarding the localization, pattern of distribution, and clinical and histopathological features of the lesions

\begin{tabular}{|l|l|}
\hline & $\begin{array}{l}\text { Number } \\
\text { (total = 26) }\end{array}$ \\
\hline Localization & \\
\hline OD/OS & $11 / 15$ \\
\hline Bulbar & 24 \\
\hline Fornix & 1 \\
\hline Caruncle & 1 \\
\hline Limbal involvement & 15 \\
\hline Clinical features & \\
\hline Nodular & 12 \\
\hline Pterygium like & 7 \\
\hline Gelatinous & 6 \\
\hline Caruncle mass & 1 \\
\hline Pattern of distribution & \\
\hline Nasal & 15 \\
\hline Temporal & 7 \\
\hline Superior & 2 \\
\hline Inferior & 1 \\
\hline Histopathological features & \\
\hline Cornea/conjunctival intraepithelial neoplasia & 10 \\
\hline SCC & 15 \\
\hline Caruncle carcinoma & 1 \\
\hline & \\
\hline
\end{tabular}

\section{Discussion}

OSSN typically arises adjacent to the limbus, over a preexisting pinguecula; that is, over an area of solar elastosis.

The purpose of the present retrospective case series is to show diverse clinical presentation of OSSN with a rare presentation of caruncle SCC.

The age and sex distributions of OSSN patients in this study were consistent with those of prior studies; ${ }^{6}$ a majority of cases were presented during the fifth to seventh decades with a mean age of 54.2 years and with marked male predominance (69\%). Male predominance is justified by the climatic and occupational issues of patients involving outdoor work and more ultraviolet light exposure. 


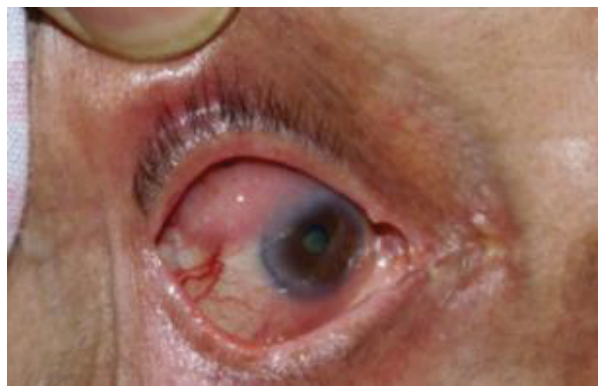

Fig. 3. Diffuse and large OSSN.

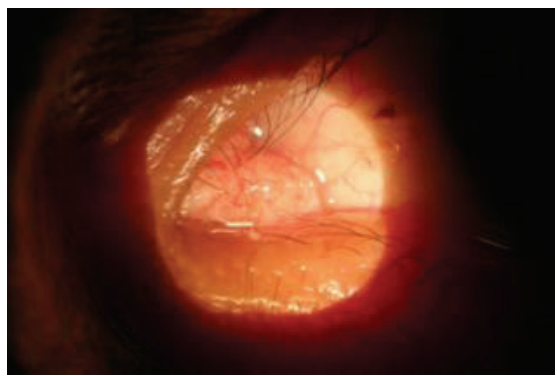

Fig. 4. Caruncle carcinoma presented with asymptomatic medial canthal mass.

Clinically, OSSN has a high presentation rate with different growth patterns. In this regard, our results are consistent with other studies, ${ }^{3}$ which reported the most common morphological growth pattern to be nodular (Fig. 1).

A significantly higher number of SCC in bulbar conjunctiva has been reported in the nasal part compared with the temporal part, which is in line with the results of a previous study.

We reported a rare case of primary squamous carcinoma of caruncle (Fig. 4), which was resected completely followed by the topical chemotherapy and showed total cure with no recurrence during a 20-month follow-up.

Because of the inherent difficulty in clearly differentiating dysplasia, carcinoma in situ, and SCC based solely on clinical features, clinicians often tend to use the generic diagnosis of OSSN. ${ }^{8}$

Shields et al. identified that the greatest relative risk for SCC versus cervical intraepithelial neoplasia included a diffuse configuration, brown pigmentation, $>10 \mathrm{~mm}$ median basal diameter, and $>1 \mathrm{~mm}$ thickness. ${ }^{8,9}$

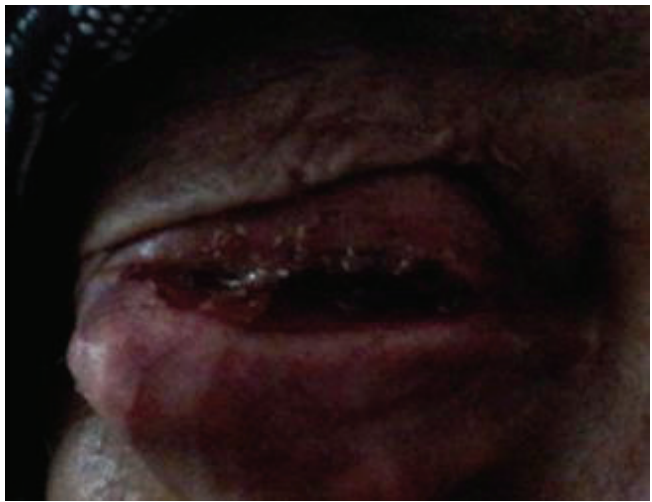

Fig. 5. Recurrent OSSN with orbital extension. 
In a study conducted to evaluate the recurrence rate of OSSN after excision and cryotherapy, Li et al. showed a recurrence rate of $7.1 \% .{ }^{10}$

In our study, 24 patients were disease free at a mean follow-up of 20 months and the recurrence rate was $7.5 \%$.

We had an orbital extension in a patient who refused further intervention after recurrence (Fig. 5). In a study by Ali et al., the most common malignancy leading to an orbital extension was an extensive OSSN. ${ }^{11}$

This study had a number of limitations including a short-term follow-up and a limited number of patients.

\section{Conclusion}

In conclusion, although OSSN has numerous clinical features, its most common presentation is a nodular type of lesion. OSSN also can be presented as a pterygium. The early suspicion of ocular surface neoplasia will be accomplished with simple excision in the most cases even in caruncle and favorable outcome except in advance case with conflict consequence.

\section{References}

1. Lee GA, Hirst LW. Ocular surface squamous neoplasia. Surv Ophthalmol. 1995;39(6):429-450.

2. Mitchael T, Carol LK. Modern management of ocular surface squamous neoplasia. Expert Rev Ophthalmol. 2013;8(3):287-295.

3. Dandala PP, Malladi P, Kavitha. Ocular Surface Squamous Neoplasia (OSSN): a retrospective study. J Clin Diagn Res. 2015;9:NC10-NC13.

4. Kao AA, Galor A, Karp CL, Abdelaziz A, Feuer WJ, Dubovy SR. Clinicopathologic correlation of ocular surface squamous neoplasms at Bascom Palmer Eye Institute: 2001 to 2010. Ophthalmology. 2012;119(9):1773-1776.

5. Atallah M, Joag M, Galor A, et al. Role of high-resolution optical coherence tomography in diagnosing ocular surface squamous neoplasia with coexisting ocular surface diseases. Ocul Surf. 2017 Oct;15(4):688-695.

6. Meel R, Dhiman R, Vanathi M, Pushker N, Tandon R, Devi S. Clinicodemographic profile and treatment outcome in patients of ocular surface squamous neoplasia. Indian J Ophthalmol. 2017;65:936-941.

7. Tanabe M, Yoshikawa H, Onishi Y, Kohno R, Ishibashi T. Ocular surface squamous neoplasia: analysis of 34 cases. Nippon Ganka Gakkai Zasshi. 2014 May;118(5):425-432.

8. Honavar SG. Ocular surface squamous neoplasia: are we calling a spade a spade? Indian J Ophthalmol. 2017;65:907-909

9. Shields $\mathrm{CL}$, Alset AE, Boal NS, et al. Conjunctival tumors in 5002 cases. Comparative analysis of benign versus malignant counterparts. The 2016 James D. Allen Lecture. Am J Ophthalmol. 2017;173:106-133.

10. Li AS, Shih CY, Rosen L, Steiner A, Milman T, Udell IJ. Recurrence of Ocular Surface Squamous Neoplasia treated with excisional biopsy and cryotherapy. Am J Ophthalmol. 2015 Aug; 160(2):213-219.

11. Ali MJ, Pujari A, Dave TV, Kaliki S, Naik MN. Clinicopathological profile of orbital exenteration: 14 years of experience from a tertiary eye care center in South India. Int Ophthalmol. 2016 Apr;36(2):253-258. 